\title{
Position-Dependent Diffusion from Biased Simulations and Markov State Model Analysis
}

\author{
Supporting Information \\ François Sicard ${ }^{1,2},{ }^{*}$ Vladimir Koskin ${ }^{1,2}$, Alessia Annibale ${ }^{3}$, and Edina Rosta ${ }^{1,2 \dagger}$ \\ 1 Department of Chemistry, King's College London, SE1 1DB London, UK \\ 2 Department of Physics and Astronomy, University College London, WC1E 6BT London, UK and \\ 3 Department of Mathematics, King's College London, SE11 6NJ London, UK
}

\section{1D Langevin Dynamics}

Analytical Potentials. In the model systems evolving under the Brownian overdamped and the full inertial limit of the Langevin equation in 1D, the deterministic force, $F(x)=-\nabla V(x)$, in Eqs. 14 and 18 in the main text derives from the potential energy $V(x)=V_{\text {ref }}(x)+V_{\text {bias }}(x)$, where $V_{\text {ref }}=\sum_{n=0}^{6} \alpha(n) x^{n}$ is defined as a polynomial of degree 6 , with parameters $\alpha(n)$ given below for the low $\left(V_{\text {low }}\right)$ and high $\left(V_{\text {high }}\right)$ energy barrier potentials:

\begin{tabular}{c|c|c|c|c|c|c|c}
\hline$V_{\text {ref }}$ & $\alpha(0)$ & $\alpha(1)$ & $\alpha(2)$ & $\alpha(3)$ & $\alpha(4)$ & $\alpha(5)$ & $\alpha(6)$ \\
\hline \hline$V_{\text {low }}$ & 22.7498 & -301.374 & $1.3865 \times 10^{3}$ & $-2.9683 \times 10^{3}$ & $3.2173 \times 10^{3}$ & $-1.7111 \times 10^{3}$ & 354.3680 \\
\hline$V_{\text {high }}$ & 47.2598 & -722.6061 & $3.8067 \times 10^{3}$ & $-8.7222 \times 10^{3}$ & $9.7780 \times 10^{3}$ & $-5.2710 \times 10^{3}$ & $1.0919 \times 10^{3}$ \\
\hline
\end{tabular}

The biased energy potential, $V_{\text {bias }}=\frac{1}{2} K\left(x-x^{(k)}\right)^{2}$ is defined as an harmonic potential with $K$ the biasing spring constant and $x^{(k)}$ the center of the harmonic bias in simulation $k$, uniformly distributed along the reaction coordinate $(\mathrm{RC})$.

Analytical Diffusion coefficients. We considered either a quadratic or step-like position-dependent diffusion profile, $D(x)=k_{B} T / \gamma(x)$ defined by the generalization of Einstein's relation, with $k_{B}$ the Boltzmann constant, $T$ the temperature of the system, and $\gamma(x)$ the position-dependent friction defined as a parabolic $(\mathrm{P})$ or a $Z$-shaped membership (Z) function,

$$
\begin{aligned}
& \gamma^{P}(x)=\gamma_{0}^{P}\left(1-\frac{1}{3}\left(x-x_{P}\right)^{2}\right), \\
& \gamma^{Z}(x)=\gamma_{0}^{Z}(2+\operatorname{zmf}(x, a, b)),
\end{aligned}
$$

with $\gamma_{0}^{P}=3000, x_{P}=0.8, \gamma_{0}^{Z}=1700, a=0.5, b=1.1$. The function $\operatorname{zmf}(x, a, b)$ represents the sigmoidal membership function defined as

$$
\operatorname{zmf}(x, a, b)= \begin{cases}1, & x \leq a \\ 1-2\left(\frac{x-a}{b-a}\right)^{2}, & a \leq x \leq \frac{a+b}{2} \\ 2\left(\frac{x-b}{b-a}\right)^{2}, & \frac{a+b}{2} \leq x \leq b \\ 0, & x \geq b\end{cases}
$$

\footnotetext{
* Corresponding author: francois.sicard@free.fr.

$\dagger$ Corresponding author: edina.rosta@kcl.ac.uk.
} 
Biased 1D Langevin dynamics in $V_{\text {high }}$. We considered the biased evolution of the system in the high $\left(V_{\text {high }}\right)$ barrier potential defined above, within the umbrella sampling framework. We studied the effect of increasing the biasing spring constant, $K$, from $\sim 800 k_{B} T$ to $\sim 3000 k_{B} T$, on the reconstruction of the diffusion profile. We first evaluated the second conditional moments $c^{(2)}$, defined in Eq. 4 in the main text, as shown in Fig. S1. The diffusion coefficient was subsequently assessed from the slope of a weighted polynomial regression. As shown in Fig. S2, we observed excellent agreement between the theoretical profiles and the numerical results. For the lowest biasing spring constant, $K \sim 800 k_{B} T$, we oberved higher variability around the transition states $X \approx 0.5$ and $\approx 1.2$, as shown in Fig. S2 (a). Increasing the biasing spring constant, $K$, from $\sim 800 k_{B} T$ to $\sim 3000 k_{B} T$, yields better sampling around the transition states, with lower variability on the reconstruction of the diffusion profile around the transition states, as shown in Fig. S2 (b).

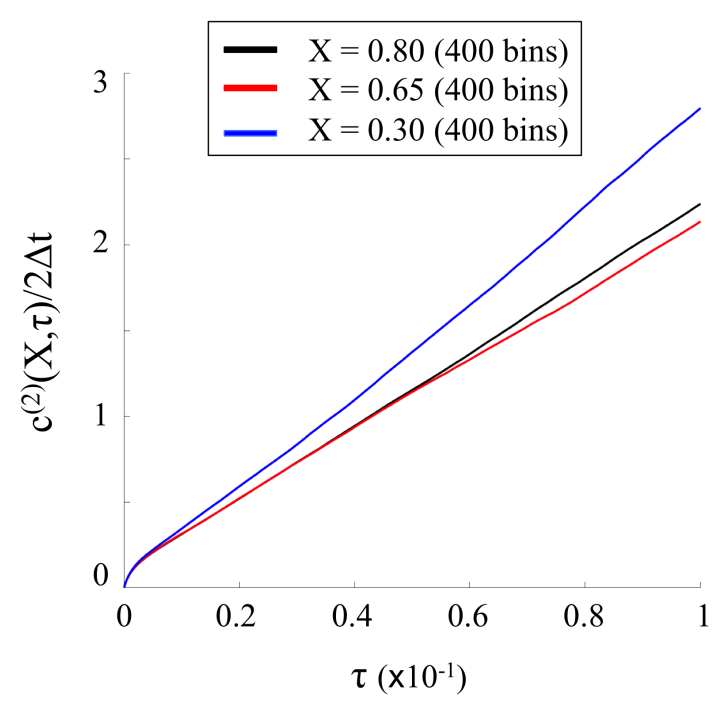

FIG. S1. Evolution of $c^{(2)}(X, \tau)$ in the biased full inertia Langevin dynamics and $V_{\text {high }}$ for three different position along the RC. The discretization of the system considered here was $N_{\text {bin }}=400$.

(a)

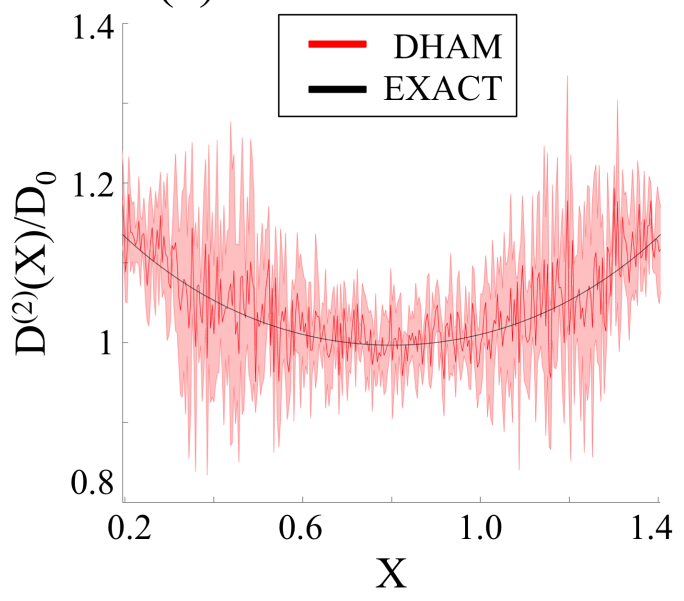

(b)

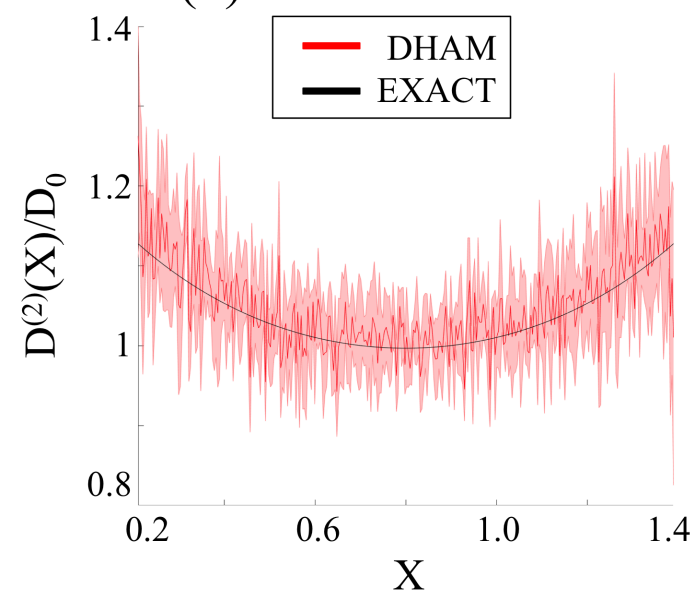

FIG. S2. Diffusion profiles obtained with biased full inertia Langevin simulations in $V_{\text {high }}$ and quadratic diffusion when the biasing spring constant $K \sim 800 k_{B} T$ (a) and $K \sim 3000 k_{B} T$ (b). The Diffusion coefficient $D^{(2)}(X)$ is rescaled to $D_{0}=k_{B} T / \gamma_{0}^{P}$ for clarity. The discretization of the system considered here was $N_{\text {bin }}=400$. The profiles obtained within the DHAM framework (red) agree within standard error with the exact profiles obtained from the generalization of Einstein's relation (black). Uncertainties, as represented in shaded area, were estimated from 10 independent runs, determining the profiles independently, and calculating the standard error. 


\section{2D Langevin Dynamics}

Analogously to the $1 \mathrm{D}$ case, we constructed a finely discretized 2D grid to determine the MSMs along the two RCs, $X$ and $Y$, using the 2D-DHAM method. We considered a 2D binning of $90 \times 90$, sufficiently small to measure accurately the diagonal elements of the diffusion tensor, $D_{11}$ and $D_{22}$, shown in Fig. 6 in the main text. To do so, we first evaluated the diagonal conditional moments, $c_{X X}$ and $c_{Y Y}$, defined in Eq. 13 in the main text, as shown in Figs. S3 (a) and (b), respectively. The diffusion coefficient was subsequently assessed from the slope of a weighted polynomial regression.

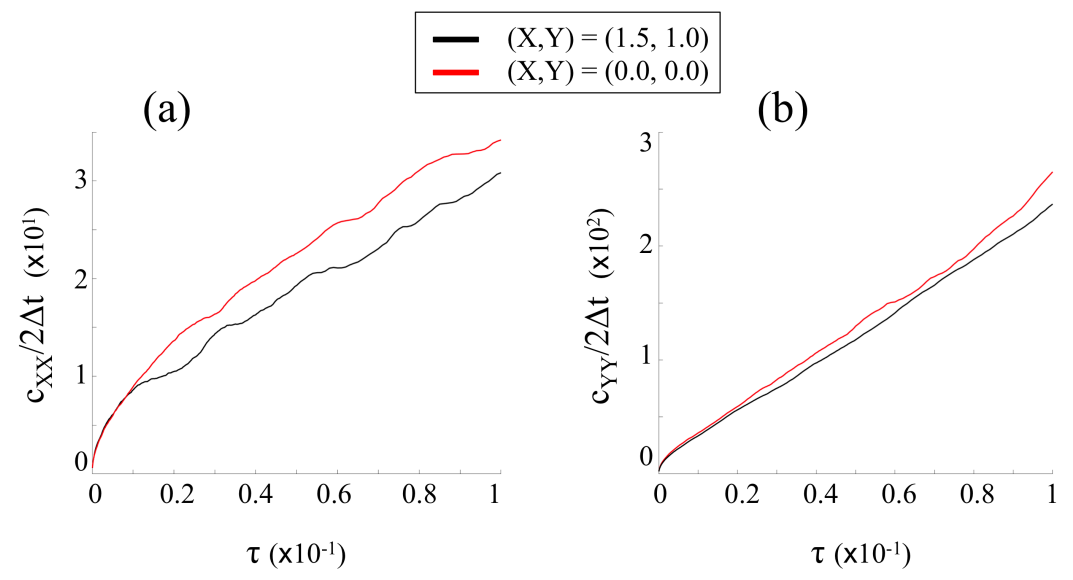

FIG. S3. Evolution of the diagonal conditional moments $c_{X X}^{(2)}(\mathrm{a})$ and $c_{Y Y}^{(2)}$ (b) in the biased Brownian overdamped Langevin dynamics in $2 \mathrm{D}$, for two different positions in the potential energy surface $(X, Y)=(1.5,1.0)$ and $(X, Y)=(0,0)$ and $N_{\text {bin }}=$ $90 \times 90$.

\section{Water-mediated conformations of Alanine Dipeptide in 1D}

To obtain the diffusion profile associated with the conformational transition between the metastable states $\alpha$ and $\beta$ of ALA2 shown in Fig. 7 in the main text, we assessed the Markov timescale $\tau_{M}$ associated with the MD trajectory for a discretization $N_{\text {bin }}=1000$ (Fig. S4 (a)). We compared our results with those obtained with a different discretization $N_{\text {bin }}=500$, which did not show significant changes (data not shown). We then evaluated the second conditional moments, $c^{(2)}$, over the range of lagtime $\tau \geq \tau_{M}$, as shown in Fig. S4 (b). The diffusion coefficient was assessed from the slope of a weighted polynomial regression. We calculated the fourth-order coefficient and check the ratio $D^{(4)}(X) /\left(D^{(2)}(X)\right)^{2}<10^{-2}$, indicating the necessary condition of the Pawula theorem holds. We also compared the force profile, $F(\Psi)$, obtained from the gradient of the potential of mean force calculated with DHAM with $F(\Psi)$

(a)

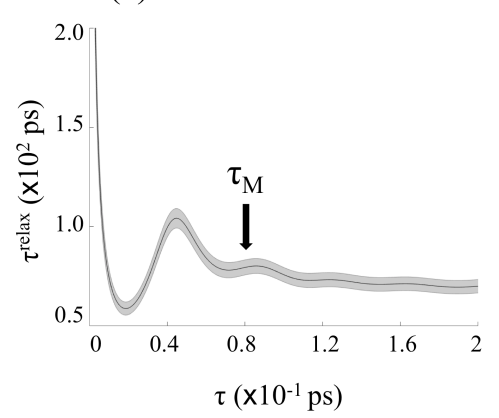

(b)

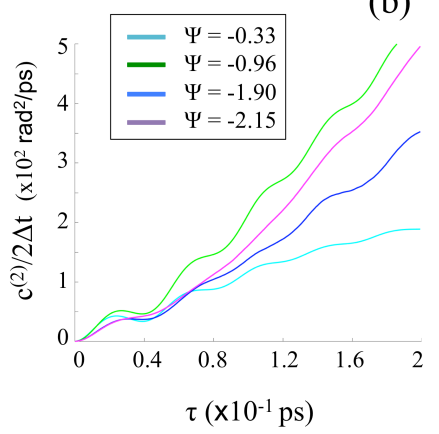

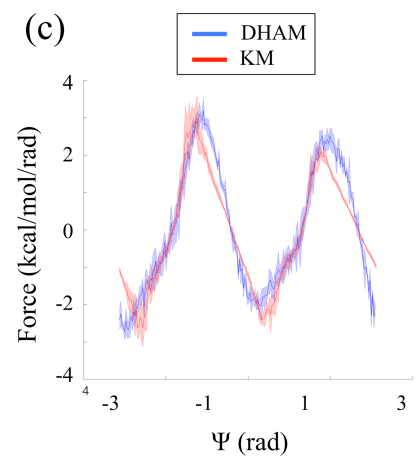

FIG. S4. Evolution of (a) the slowest relaxation time, $\tau^{\text {relax }}$, and (b) the conditional moment $c^{(2)}$ for different values of the dihedral angle $\Psi$ measured for $N_{\text {bin }}=1000$. (c) Force profiles obtained from DHAM and the KM expansion. Uncertainties, as represented in shaded area, were estimated from 3 independent runs, determining the profiles independently, and calculating the standard error. 
obtained from the KM expansion $\left(k_{B} T D^{(1)}(\Psi) / D^{(2)}(\Psi)\right)$, as shown in Fig. S4 (c). The 2 profiles show good numerical agreement, providing additional self-consistency using our method.

\section{Membrane permeation}

To obtain the 1D diffusion profile associated with the permeation of Domperidone, a dopamine receptor antagonist, across the lipid (POPC) membrane shown in Fig. 8 in the main text, we first assessed the Markov timescale $\tau_{M}$ associated with the MD trajectory for a discretization $N_{\text {bin }}=1000$ (Fig. S5 (a)). The relaxation time can be seen to level off in the region of lagtimes greater than $100 \mathrm{ps,} \mathrm{where} \mathrm{it} \mathrm{is} \mathrm{sufficiently} \mathrm{large} \mathrm{for} \mathrm{the} \mathrm{relaxation} \mathrm{time} \mathrm{to} \mathrm{be}$ insensitive to the precise choice of the lagtime, as shown in Fig. S5 (a). We then evaluated the second conditional moments $c^{(2)}$ defined in Eq. 4 in the main text over the range of lagtime $\tau \geq \tau_{M}$, as shown in Fig. S5 (b). The diffusion profile, as shown in Fig. S6, was subsequently assessed from the slope of a weighted polynomial regression. We calculated the fourth-order coefficient and check the ratio $D^{(4)}(X) /\left(D^{(2)}(X)\right)^{2}<10^{-2}$, indicating the necessary condition of the Pawula theorem holds (data not shown).

Analogously to the $1 \mathrm{D}$ case, we constructed a finely discretized 2D grid to determine the MSMs along two reaction coordinates, $Z$ and $\Delta z$, using the 2 D-DHAM method. We considered a $2 \mathrm{D}$ binning of $50 \times 50$, sufficiently small to evaluate the second conditional moments (see Fig. S7), and measured the diagonal and off-diagonal elements of the diffusion tensor in the localized area defined in Fig. 9 in the main text. We calculated the fourth-order coefficient and check the ratio $D_{i i}^{(4)}(X) /\left(D_{i i}^{(2)}(X)\right)^{2}<2 \times 10^{-2}$, indicating the necessary condition of the Pawula theorem holds.

(a)

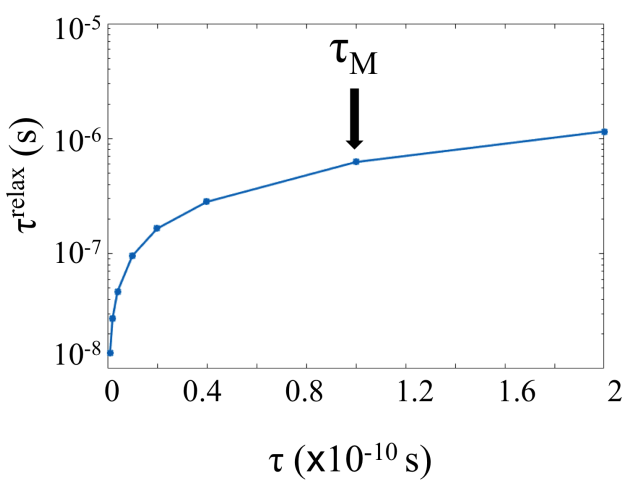

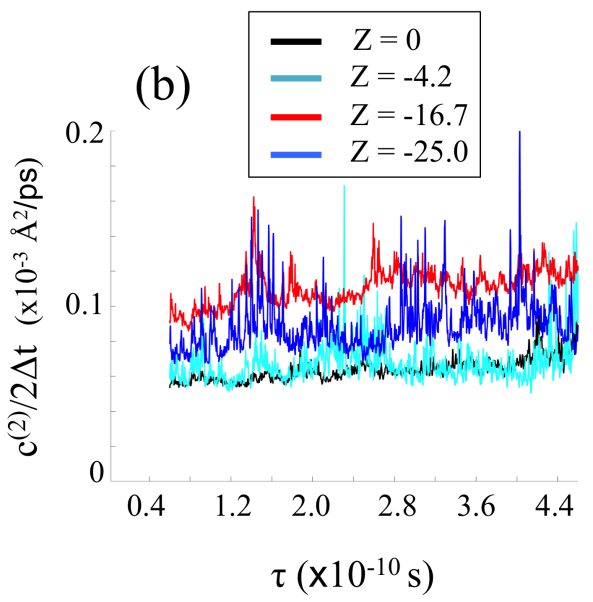

FIG. S5. Evolution of (a) the slowest relaxation time, $\tau^{\text {relax }}$, and (b) the conditional moment $c^{(2)}$ for different values of the position $Z$ of the ligand through the POPC membrane measured for $N_{\text {bin }}=1000$. 


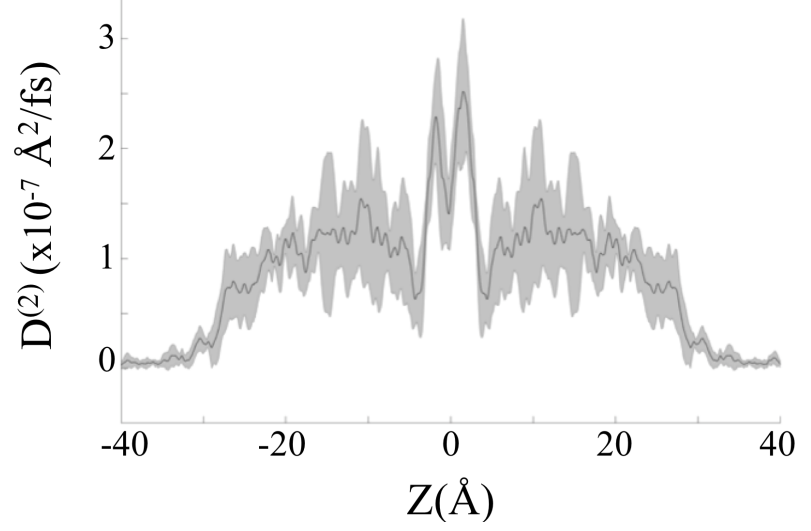

FIG. S6. Diffusion profile associated with the crossing of Domperidone through the (POPC) bilayer and reconstructed along the distance from the center of the bilayer, $Z$, measured for $N_{\text {bin }}=1000$. Uncertainties, as represented in shaded area, were determined by dividing the data into two equal sections, determining the profiles independently, and calculating the standard error.

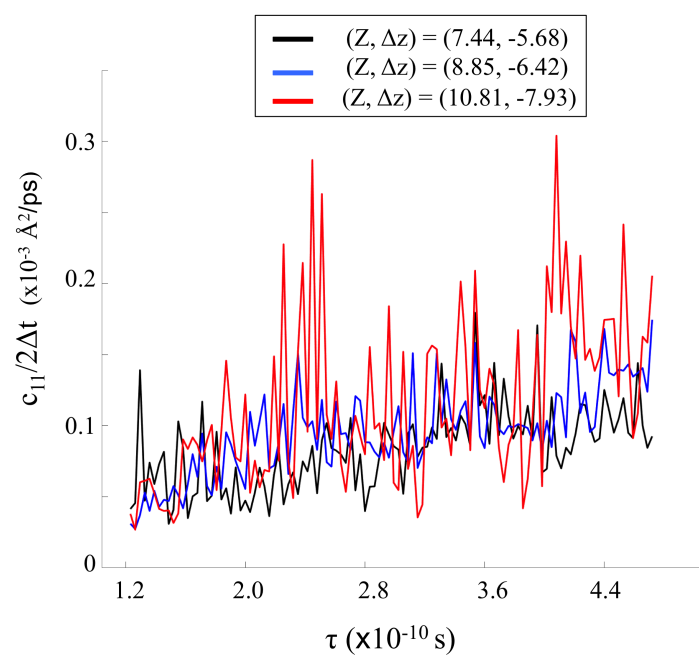

FIG. S7. Evolution of the diagonal conditional moments $c_{11}^{(2)}$ obtained in the analysis of the membrane permeation in $2 \mathrm{D}$ for three different positions in the potential energy surface $(Z, \Delta z)$ and $N_{\text {bin }}=50 \times 50$. 